\title{
Veteran Suicide Risk Factors: A National Sample of Nonveteran and Veteran Men Who Died by Suicide
}

\author{
David S. Wood \\ Brigham Young University - Provo, d_wood@byu.edu \\ Bethany M. Wood \\ University of Texas at Austin \\ Aislinn Watson \\ Devan Sheffield \\ Rocky Vista University \\ Helena Hauter \\ Utah State Hospital \\ Follow this and additional works at: https://scholarsarchive.byu.edu/facpub \\ Part of the Social Work Commons
}

\section{Original Publication Citation}

David S Wood, Bethany M Wood, Aislinn Watson, Devan Sheffield, Helena Hauter, Veteran Suicide Risk Factors: A National Sample of Nonveteran and Veteran Men Who Died by Suicide, Health \& Social Work, Volume 45, Issue 1, February 2020, Pages 23-30.

\section{BYU ScholarsArchive Citation}

Wood, David S.; Wood, Bethany M.; Watson, Aislinn; Sheffield, Devan; and Hauter, Helena, "Veteran Suicide Risk Factors: A National Sample of Nonveteran and Veteran Men Who Died by Suicide" (2020). Faculty Publications. 4112.

https://scholarsarchive.byu.edu/facpub/4112

This Peer-Reviewed Article is brought to you for free and open access by BYU ScholarsArchive. It has been accepted for inclusion in Faculty Publications by an authorized administrator of BYU ScholarsArchive. For more information, please contact ellen_amatangelo@byu.edu. 


\title{
Veteran Suicide Risk Factors: A National Sample of Nonveteran and Veteran Men Who Died by Suicide
}

\author{
David S. Wood, Bethany M. Wood, Aislinn Watson, Devan Sheffield, and Helena Hauter
}

\begin{abstract}
Veteran suicide is a serious public health problem. Some data suggest that veteran suicide risk profiles differ from those of nonveterans. Records for veteran $(n=21,692)$ and nonveteran $(n=83,430)$ men who died by suicide were examined from 17 U.S. states using the National Violent Death Reporting System data. Seventeen precipitating factors were examined and combined through meta-analysis of proportions. Many precipitating factors were found to be less frequent for veterans. A smaller number of factors were found to be higher in the veteran population, including physical health problems. A sizable cumulative effect size (1.02) was observed, suggesting that veteran and nonveteran men show meaningful and substantive differences in their risk profiles-differences that should be considered when planning and implementing suicide prevention and intervention efforts. The conspicuous role of physical health problems among veterans who die by suicide is discussed. The article concludes with specific practice recommendations for social workers.
\end{abstract}

KEY WORDS: National Violent Death Reporting System; physical health; predictors; suicide; veterans

\section{$\mathrm{T}$}

he topic of veteran suicide is of critical importance in the United States. Veterans have typically had higher rates of suicide than the general population (McCarthy et al., 2015), and these rates appear to be increasing (U.S. Department of Veteran Affairs [VA], 2018). It is estimated that in the United States, approximately 22 veterans die by suicide per day (VA, 2018). Although post-suicide intervention is a significant point of intervention among family members and communities that have lost an individual to suicide, finding predictive variables that increase the risk of suicide among individuals can inform prevention efforts. Identifying risk factors is a salient approach to prevent suicide among military veterans in the United States because most studies demonstrate that veterans are more likely than nonveterans to end their own life (Kaplan, McFarland, Huguet, \& Valenstein, 2012; Miller, Azrael, Barber, Mukamal, \& Lawler, 2012). If predictive risk factors are identified, then the individuals most at risk can benefit from suicide intervention approaches such as psychotherapy.

When examining predictive factors associated with suicide, current methods suggest that inter- vention consists of getting subjective input from the person at risk. One approach uses the Columbia Suicide Severity Rating Scale, which elicits input regarding suicide ideation, intention of suicide, planning, and history of suicidal behavior as a method to intervene with a potential victim of suicide (Hill, Hatkevich, Kazimi, \& Sharp, 2017). This approach has been shown to be effective (Kim, Jeong, \& Kim, 2017). Although direct intervention (that is, asking about suicide) is often effective, this approach relies on the subjective report of the individual and assumes a high level of candor from the person at risk of suicide. Identifying objective predictive factors also has an important place in suicide prevention. Distinguishing empirically derived precipitating circumstances among actual suicide deaths in addition to direct intervention could increase the accuracy of identifying people at risk and increase the effectiveness of suicide intervention. More research is needed in this area.

We examined precipitating circumstances among individuals who died by suicide. Suicide prevention and intervention may differ for veterans and nonveterans in the United States based on mil- 
itary service and experiences. The present study aids in addressing the question of divergent suicide risk profiles of veteran and nonveteran men by examining a large U.S. data set.

\section{VETERAN SUICIDE}

The prevalence of death by suicide has been on the rise in the United States since 1999 (Curtin, Warner, \& Hedegaard, 2016), so that suicide is now one of the top 10 leading causes of death (Kochanek, Murphy, Xu, \& Arias, 2014). With these increases among the general population, it is critical to examine suicide risk factors as a means of increasing preventive efforts for the entire population. Suicide risk is not equally distributed across all populations. This is particularly true for veteran populations.

\section{Background}

Suicide death among veterans has been examined in many studies with differing results. Undisputed is the rising trend for veteran suicide as many studies report increases in suicide risk, attempts, and death by suicide among veterans in general (McCarthy et al., 2015) and those actively serving in the military (Hoge \& Castro, 2012; Hom, Stanley, Gutierrez, \& Joiner, 2017; Nock et al., 2014; Ursano et al., 2015). The actual rate of U.S. military service members who died by suicide exceeded the rate for those in the general population in 2009 (Dao \& Lehren, 2013). Prior to the rise in the rates of suicide among veterans in the United States, a common assumption was that the rigorous selection process of the military would screen out any potentially suicidal individuals; however, due to the increased suicide rate, this assumption no longer holds true (Bruce, 2010; Nock et al., 2014) and specific risk factors that are unique to veterans are emerging in the research literature. Substance use disorders have been shown to be a highly salient risk factor for veterans as compared with nonveterans (Ilgen et al., 2010). The preponderance of veterans in rural environments likely enhances the suicide risk factor of social isolation and thwarted belongingness (McCarthy et al., 2012).

Distinctive suicide risk factors for veterans have been profitably integrated in clinical treatment approaches that have been shown to be effective for reducing suicide risk with military service members and veterans, including collaborative approaches
(Huh et al., 2018) and suicide-specific interventions (Bryan \& Rudd, 2018). The VA and the Department of Defense have implemented a range of public health-informed approaches to suicide prevention, including group therapy, that have the potential to mitigate thwarted belongingness among veterans who are at risk of suicide (Johnson, O'Connor, Kaminer, Jobes, \& Gutierrez, 2014). A veteran-focused view of suicide prevention can maximize and enhance brief screening and referral programs for veterans at risk of suicide, such as that provided in emergency departments (Stanley et al., 2015).

\section{Suicide Risk Theory among Veterans}

Although the risk factors of the general population apply to military veterans, much of the literature has focused on differentiating unique risks found within the latter population. The interpersonalpsychological theory of suicide (IPTS) has been used to understand veteran suicide through a focus on its three tenets - that suicidal behavior is predicted by thwarted belongingness, perceived burdensomeness, and acquired capability (Griffith \& Vaitkus, 2013; Joiner et al., 2009) in the United States. Another theory of suicide called fluid vulnerability theory (FVT) proposes that suicide risk is neither linear nor static and that it often spikes rapidly based on shifting psychological and physical aspects of individuals (Rudd, 2006). Wolfe-Clark and Bryan (2016) suggested that both theories provide a helpful predictive and explanatory lens for understanding suicide risk. In particular, FVT and IPTS may help explain the role of physical health problems among veterans at risk of suicide. Military service members serve in a culture that prizes physicality but one that also presents a high rate of physical injuries, which can disrupt physical functioning (Knapik et al., 2013). This is augmented by research suggesting that veterans may be less likely to seek mental health counseling when they have a serious health problem, thus compounding the risk for suicide and other mental health repercussions (Levine et al., 2016).

\section{CORE RESEARCH QUESTIONS}

To better understand suicide risk among veterans, our study sought to address the following two research questions: (1) What unique risk factors for suicide are found among male veterans as compared with men from the general U.S. population? 
and (2) What is the overall effect of military veteran status on suicide risk in comparison with the risk of the general U.S. population?

\section{METHOD}

\section{Data}

This study identified specific risk factors for veterans who die by suicide using the National Violent Death Reporting System (NVDRS) derived from the Web-Based Injury Statistics Query and Reporting System through the Centers for Disease Control and Prevention (CDC) (CDC, 2017). The NVDRS is an ongoing public health program sponsored by the CDC and is a database that tracks all violent deaths, including suicide, in participating states. It is the first state database to pool data from multiple sources (that is, law enforcement, medical examiners, toxicology reports, and death certificates) about violent deaths. We examined all publicly available NVDRS data for veteran and nonveteran men from 17 states for the years 2003 to 2015 (CDC, 2015).

Data were collected through a review of all suicide deaths within each participating state and was compiled into one comprehensive data set. According to NVDRS procedures, to be included in the data set, the required documents for the deceased individual are as follows: death certificate, law enforcement reports, coroner/medical examiner records (including toxicology reports), and data abstractor input (CDC, 2015). The data are imported through electronic methods or are entered manually, if necessary. The data set is a valuable source of information to assess many mental health and social issues within the United States to guide prevention and intervention efforts (Powell et al., 2006).

\section{Sample}

We examined all publicly available data from the NVDRS for veteran and nonveteran men from 17 states, namely Alaska, Colorado, Georgia, Kentucky, Maryland, Massachusetts, New Jersey, New Mexico, North Carolina, Ohio, Oklahoma, Oregon, Rhode Island, South Carolina, Utah, Virginia, and Wisconsin. We excluded Michigan, because data were only available for one year. Both veteran and nonveteran men were selected as the unit of analysis for this article because men are more likely to die by suicide and by different means than women in the general U.S. population (Olfson et al., 2017); in the military there are higher enlistments from men and the current active-duty force consists of 85.5 percent men (Watkins, 2017). In addition, the NVDRS presents very low sample sizes of female veterans who died by suicide. Therefore, future studies will investigate both men and women as veterans with risk of suicide. The years of analysis were based on when data are available in the 17 states, the earliest being 2001 and the most recent being 2015 (CDC, 2015). Veteran status was coded when the decedent had previously served in the U.S. armed forces, including all the branches: Army, Navy, Air Force, Marines, and Coast Guard. Military service includes active, reserve, and National Guard.

\section{Variables}

The independent variable in this analysis was veteran status, and the dependent variables were 18 precipitating factors that are recorded in the NVDRS. We excluded the variable "school problem" due to a zero count for veterans in most states in the database. Deaths from chronic substance abuse, acute substance-induced deaths that lack evidence of intent of self-harm, legal assisted suicides, and deaths resulting from autoerotic behavior were not coded as suicides in the NVDRS (CDC, 2015).

Seventeen different precipitating factors were used to assess the risk of suicide and used as the dependent variables, including (1) current depressed mood, (2) leaving a suicide note, (3) current mental health problem, (4) disclosure of intent to die by suicide, (5) current treatment for mental illness, (6) history of suicide attempts, (7) past treatment for mental illness, (8) a recent crisis, (9) intimate partner problem, (10) other relationship problems, (11) eviction or loss of home, (12) alcohol dependence, (13) other substance abuse problems, (14) recent criminal or legal problems, (15) other legal problems, (16) physical health problems, and (17) death of a friend or family member. In the past five years (see Table 1 for a list and operational definition of these factors).

\section{Analyses}

Our primary analyses included computing proportions, combining proportions through metaanalysis, comparing meta-analyzed proportions using binomial z-tests and computing an index of the cumulative effect size attributable to veteran status. We explain each step here. 


\section{Table 1: NVDRS Risk Factors and Other Precipitating Circumstances Variable Codebook}

NVDRS Variable

Current depressed mood

Leaving a suicide note

Current mental health problem

Disclosed intent to die by suicide

Treatment for mental illness

History of suicide attempts

Past treatment for mental illness

Crisis during the past two weeks

Intimate partner problem

Other relationship problems

Eviction or loss of home

Alcohol dependence

Other substance abuse problems

Recent criminal legal problems

Other legal problems

Physical health problems

Death of friend or family during

the past five years
Victim was perceived by self or others to be depressed at the time of the injury.

Victim left a suicide note (or other recorded communication). Note can be written or electronic.

Current mental health problem (includes those listed in the DSM-5).

Victim disclosed to another person their thoughts or plans to commit suicide within the last month. Disclosure of suicidal thoughts or plans can be verbal, written, or electronic.

Current treatment for mental illness or substance abuse problem.

Victim has a history of attempting suicide before the fatal incident.

History of ever being treated for mental health or substance abuse problem.

Victim experienced a crisis within two weeks of the incident, or a crisis was imminent within two weeks of the incident that was not associated with another circumstance variable.

Problems with a current or former intimate partner appear to have contributed to the suicide or undetermined death.

Problems with a friend or an associate (other than an intimate partner or family member) appear to have contributed to the death.

A recent eviction or other loss of the victim's housing, or the threat of it, appears to have contributed to the death.

Person has alcohol dependence or alcohol problem.

Person has a nonalcoholic-related substance abuse problem.

Criminal legal problems appear to have contributed to the death.

Civil legal (noncriminal) problems appear to have contributed to the death.

Victim's physical health problems appear to have contributed to the death.

Suicide of a family member or friend appears to have contributed to the death

Note: NVDRS = National Violent Death Reporting System.

First, we examined 18 predictive risk factors that are recorded in the NVDRS (for example, depressed mood, leaving a suicide note, physical health problems). Then we computed the proportion of veteran decedents who exhibited each predictive risk factor across years for each state.

Second, we combined these proportions across years using meta-analysis using Stata 15 with the Metaprop program developed by Nyaga and Arbyn (2014). The Metaprop program uses the FreemanTukey double arcsine transformation of proportions. We used a random-effects model to account for heterogeneity in the data. At this stage we also combined the proportion of veteran suicide deaths for all states into a final statistic for each risk factor. We did not analyze any moderators or possible dependencies. We used listwise deletion for each analysis of every precipitating factor for a given year, by excluding any state that had missing values. We did not use any imputation method.

Third, the final proportions for each risk factor were tested against the overall proportion of veteran suicide deaths using a binomial $\mathrm{z}$ test in Stata 15 . The overall proportion of veteran suicide deaths was 26 percent (veteran $=21,692$ deaths; nonveteran $=83,430$ deaths). As an example, the proportion of male veteran suicide decedents across all states who had a current mental health problem was 24 percent. The binomial $\mathrm{z}$ test revealed a significant deviation from the expected proportion of 26 percent $(p<.001)$. Statistically significant risk factors that were lower than expected are shown in Table 2, and those that were higher than expected are shown in Table 3.

Due to the large sample size, most of the findings were statistically significant - therefore our results focus more on the effect sizes, which yield a practical estimation of effect. Effect-size statistics were calculated by subtracting the observed proportion for each predictive factor from the expected or hypothesized proportion (that is, null hypothesis) (Green \& Salkind, 2016). The total effect size attributable to veteran status was calculated by summing the absolute value for each significant 
Table 2: Lower than Expected Proportions of Male Veteran Suicide Deaths across All States by Precipitating Circumstance

\begin{tabular}{|c|c|c|c|c|c|c|c|c|c|c|}
\hline \multirow[b]{2}{*}{ Precipitating Circumstance } & \multicolumn{4}{|c|}{ Military } & \multicolumn{4}{|c|}{ Nonmilitary } & \multirow{2}{*}{$\begin{array}{c}\text { Test of } \\
\text { Proportion } \\
\text { for Militar } \\
(p)^{a}\end{array}$} & \multirow[b]{2}{*}{$\mathbf{E S}^{\mathbf{b}}$} \\
\hline & $\%$ & $\mathbf{L C I}$ & $\mathbf{U C I}$ & $n$ & $\%$ & $\mathbf{L C I}$ & UCI & $n$ & & \\
\hline Current depressed mood & .25 & .24 & .27 & 8,004 & .75 & .73 & .76 & 23,193 & .084 & -.01 \\
\hline Current mental health problem & .25 & .23 & .26 & 7,272 & .75 & .74 & .77 & 22,765 & $.000^{*}$ & -.01 \\
\hline Current treatment for mental illness & .24 & .23 & .26 & 5,107 & .76 & .74 & .77 & 16,091 & $.000^{*}$ & -.02 \\
\hline Ever treated for mental problem & .24 & .22 & .25 & 6,044 & .76 & .75 & .78 & 19,701 & $.000^{*}$ & -.02 \\
\hline Disclosed intent to commit suicide & .24 & .22 & .26 & 5,263 & .76 & .74 & .78 & 16,422 & $.048^{*}$ & -.02 \\
\hline History of suicide attempts & .20 & .18 & .21 & 2,417 & .80 & .79 & .82 & 9,823 & $.000^{*}$ & -.06 \\
\hline Crisis in past two weeks & .23 & .21 & .25 & 5,487 & .77 & .75 & .79 & 18,424 & $.000^{*}$ & -.03 \\
\hline Intimate partner problem & .20 & .18 & .21 & 4,764 & .80 & .79 & .82 & 19,446 & $.000^{*}$ & -.06 \\
\hline Eviction or loss of home & .23 & .20 & .25 & 477 & .77 & .75 & .80 & 1,587 & $.001^{*}$ & -.03 \\
\hline Other relationship problems & .18 & .16 & .19 & 1,062 & .82 & .81 & .84 & 4,695 & $.000^{*}$ & -.08 \\
\hline
\end{tabular}

Notes: $\mathrm{LCl}=$ lower confidence interval; $\mathrm{UCI}=$ upper confidence interval.

a Tests of proportions are based on the hypothesis test of the overall proportion of male veteran suicides $(0.26)$.

${ }^{\mathrm{b}} \mathrm{ES}=$ effect size. This effect size is the difference between the observed and hypothesized (test) proportions $\left(\mathrm{P}_{\text {observed }}-\mathrm{P}_{\text {hypothesized }}\right)$. ${ }^{*} p<.05$.

\section{Table 3: Higher than Expected Proportions of Male Veteran Suicide Deaths across All} States by Precipitating Circumstance

\begin{tabular}{|c|c|c|c|c|c|c|c|c|c|c|}
\hline \multirow[b]{2}{*}{ Precipitating Circumstance } & \multicolumn{4}{|c|}{ Military } & \multicolumn{4}{|c|}{ Nonmilitary } & \multicolumn{2}{|c|}{$\begin{array}{c}\text { Test of } \\
\text { Proportion } \\
\text { for Military }\end{array}$} \\
\hline & $\%$ & $\mathbf{L C I}$ & $\mathbf{U C I}$ & $n$ & $\%$ & $\mathbf{L C I}$ & $\mathbf{U C I}$ & $n$ & $(p)^{a}$ & $\mathbf{E S}^{\mathbf{b}}$ \\
\hline Person left a suicide note & .28 & .25 & .3 & 6538 & .72 & .7 & .75 & 17618 & $.000^{*}$ & .02 \\
\hline Physical health problems & .43 & .40 & .47 & 7037 & .57 & .53 & .60 & 9434 & $.000^{*}$ & .17 \\
\hline Other death of friend or family & .30 & .28 & .33 & 1459 & .70 & .67 & .72 & 3317 & $.000^{*}$ & .04 \\
\hline
\end{tabular}

Notes: $\mathrm{LCl}=$ lower confidence interval; $\mathrm{UCI}=$ upper confidence interval.

${ }^{a}$ Tests of proportions are based on the hypothesis test of the overall proportion of male veteran suicides $(0.26)$.

${ }^{\mathrm{b}} \mathrm{ES}=$ effect size. This effect size is the difference between the observed and hypothesized (test) proportions $\left(\mathrm{P}_{\text {observed }}-\mathrm{P}_{\text {hypothesized }}\right)$ * $p<.05$.

predictive risk factor. Returning to our example predictor, current mental health problem, the effect size was $.02\left(\mathrm{P}_{\text {expected }}=0.26-\mathrm{P}_{\text {observed }}=0.24\right)$. Fourth and last, the absolute value for the effect size for each variable was summed to calculate a single value to indicate the variance of proportions that is attributable to veteran status.

\section{RESULTS}

Results from the meta-analyses indicate that most of the predictive risk factors are lower than expected for male veterans compared with the general male population of suicide decedents (see Table 2). A small number of risk factors were higher, however, than in the general population (see Table 3). For the higher than expected proportions of male veteran suicide deaths across all states by predictive risk factors, three were significantly higher: (1) person left a suicide note, (2) other death of friend or family, and (3) physical health problems. Physical health problems showed the highest deviation from the expected effect size of 0.17 ( $p$ $<.01$ ) and clearly indicated that veterans have a higher rate of physical health problems than nonveteran men. Taken together, the cumulative effect sizes were large in magnitude (observed-expected difference score $=1.02$ ). This sizable effect should not be ignored and suggests that veteran and nonveteran men show meaningful and substantive differences in their risk profiles.

\section{DISCUSSION}

Our intent in this study was to assess differences in risk profiles among veteran and nonveteran men. Indeed, the profiles of veteran and nonveteran men differ on most NVDRS variables. In short, suicide risk looks different for veterans. For a large proportion of NVDRS precipitating factors, veterans 
Table 4: Veteran-Specific Questions for Suicide Risk Assessment

Precipitating Circumstance

Physical health problems

Other death of friend or family

Suicide plan specificity (for example, suicide note)
Questions

How is your health?

Are you experiencing any physical health problems?

How does your health affect you?

How does your health make you feel?

Who of your friends or family members have died in your life?

When did this happen?

How has this affected you?

Do you have a method for suicide?

Do you have a place?

Do you have a time?

Would you write a note to your survivors?

If so, what would you say? showed lower than expected proportions whereas for a small subset of precipitating factors, veterans showed higher than expected proportions. These differences have significant ramifications for social work practice because these results may help social workers identify suicide risk factors that are unique to veterans.

In the area of suicide prevention among veterans, our findings suggest that the use of a standard male suicide risk profile would very likely lead to an underestimation of suicide risk. Veterans who are at actual risk would likely be missed and possibly experience unmitigated progression toward eventual suicide. Our findings suggest that social workers should include in their suicide risk assessments the following: (a) the person left a suicide note, (b) the recency and salience of a death of a friend or family member, and (c) the presence of a physical health problem.

\section{Suicide Note (Plan Specificity)}

It is common practice to assess whether a person at risk has made specific plans, preparations, or rehearsals for the suicide attempt. Indeed, such familiarization with a suicide attempt may contribute to increased or acquired capability for suicide (Joiner et al., 2009). Our findings suggest that veteran men who die by suicide are much more likely than nonveteran men to have left a note. We recommend that social workers assess whether a veteran has left a note or whether they intend to leave a note as a part of their contemplated or planned suicide attempt. Furthermore, Bryan and Rudd (2018) recommended asking very specific questions regarding a suicide plan (that is, method, place, time; see Table 4) rather than simply asking, "Do you have a plan?"

\section{Death of a Friend or Family Member}

Our findings suggest that veteran men who die by suicide are much more likely than nonveteran men to have had a death of friend or family member. This risk factor may contribute to what Joiner et al. (2009) termed "thwarted belongingness" and in this case, a permanent disruption in a significant relationship. We recommend that social workers assess for the recency and salience of any deaths of a friend or family member for a veteran who may be at suicide risk. Specific assessment questions are included in Table 4. Greater recency and personal impact of the death may signal increased suicide risk for veterans.

\section{Physical Health Problems}

The largest single difference in our findings between veteran men and nonveteran men who died by suicide was an increased likelihood of physical health problems among veterans. Veterans serve in military cultures that elevate physical strength and capacity. In addition, military service poses a high rate of physical injuries and disease prevalence (Knapik et al., 2013). These two opposing forces-belonging to a culture that values physical health and experiencing a high rate of physical health problems - likely contributes to a sense of perceived burdensomeness and increased suicide risk (Joiner et al., 2009). We recommend that social workers assess for the presence and severity of physical health problems when assessing for suicide risk among veterans. See Table 4 for specific ques- 
tions. Additional health assessment strategies may include the use of brief, structured health assessment questionnaires (Shamspour \& Assari, 2010).

\section{LIMITATIONS}

The present study examined only male individuals in 17 states across the United States. Further research should address suicide risk factors for women veterans. Future research should also continue to address specific kinds of physical health problems that are associated with the highest risk of suicide, using both the NVDRS and qualitative methods if necessary.

\section{CONCLUSION}

This study underscores that the suicide risk profiles of veteran men who have died by suicide differ significantly when compared with nonveteran men. Our sample was derived from 17 states across several years and promotes the importance of using veteran-specific risk factors rather than a general risk profile. Physical health problems emerged as the most distinctive difference and warrant attention as an important factor for both prevention and intervention in veteran suicide. We recommend that (a) practitioners implement questions, similar to the ones we present in this article, that address veteran-specific suicide risks (see Table 4); (b) policymakers be aware of the veteran-specific suicide risk when creating programs and resources for veterans; and (c) future researchers study the significant risks with greater focus on prevention. HSW

\section{REFERENCES}

Bruce, M. L. (2010). Suicide risk and prevention in veteran populations. Annals of the New York Academy of Sciences, 1208(1), 98-103.

Bryan, C. J., \& Rudd, M. D. (2018). Brief cognitive-behavioral therapy for suicide prevention. New York: Guilford Press.

Centers for Disease Control and Prevention. (2015). National Violent Death Reporting System: Web coding manual (Version 5.1). Retrieved from https://www .cdc.gov/violenceprevention/pdf/nvdrs_web_ codingmanual.pdf

Centers for Disease Control and Prevention. (2017). National Violent Death Reporting System. Retrieved from http://www.cdc.gov/injury/wisqars/nvdrs.html

Curtin, S. C., Warner, M., \& Hedegaard, H. (2016). Increase in suicide in the United States, 1999-2014. NCHS Data Brief, No. 241.

Dao, J., \& Lehren, A. W. (2013, May 16). In calculation of military rates, the numbers are not all straightforward. New York Times, p. A14.

Green, S. B., \& Salkind, N. J. (2016). Using SPSS for Windows and Macintosh. New York: Pearson.

Griffith, J., \& Vaitkus, M. (2013). Perspectives on suicide in the Army National Guard. Armed Forces \& Society, 39, 628-653. doi:10.1177/0095327X12471333
Hill, R. M., Hatkevich, C. E., Kazimi, I., \& Sharp, C. (2017). The Columbia-Suicide Severity Rating Scale: Associations between interrupted, aborted, and actual suicide attempts among adolescent inpatients. Psychiatry Research, 255, 338-340. doi:10.1016/j .psychres.2017.06.014

Hoge, C. W., \& Castro, C. A. (2012). Preventing suicides in US service members and veterans: Concerns after a decade of war. JAMA, 308, 671-672. doi:10.1001 /jama.2012.9955

Hom, M. A., Stanley, I. H., Gutierrez, P. M., \& Joiner, T. E., Jr. (2017). Exploring the association between exposure to suicide and suicide risk among military service members and veterans. Journal of Affective Disorders, 207, 327-335. doi:10.1016/j.jad.2016.09.043

Huh, D., Jobes, D. A., Comtois, K. A., Kerbrat, A. H., Chalker, S. A., Gutierrez, P. M., \& Jennings, K. W. (2018). The collaborative assessment and management of suicidality (CAMS) versus enhanced care as usual (E-CAU) with suicidal soldiers: Moderator analyses from a randomized controlled trial. Military Psychology, 30, 495-506. doi:10.1080/08995605.2018.1503001

Ilgen, M. A., Bohnert, A. S., Ignacio, R. V., McCarthy, J. F., Valenstein, M. M., Kim, H. M., \& Blow, F. C. (2010). Psychiatric diagnoses and risk of suicide in veterans. Archives of General Psychiatry, 67, 1152-1158.

Johnson, L. L., O’Connor, S. S., Kaminer, B., Jobes, D. A., \& Gutierrez, P. M. (2014). Suicide-focused group therapy for veterans. Military Behavioral Health, 2, 327-336. doi:10.1080/21635781.2014.963762

Joiner, T. E., Jr. Van Orden, K. A., Witte, T. K., Selby, E. A., Ribeiro, J. D., Lewis, R., \& Rudd, M. D. (2009). Main predictions of the interpersonal-psychological theory of suicidal behavior: Empirical tests in two samples of young adults. Journal of Abnormal Psychology, 118, 634-646.

Kaplan, M. S., McFarland, B. H., Huguet, N., \& Valenstein, M. (2012). Suicide risk and precipitating circumstances among young, middle-aged, and older male veterans. American Journal of Public Health, 102(Suppl. 1), S131-S137. doi:10.2105/AJPH.2011.300445

Kim, D. W., Jeong, K. Y., \& Kim, K. S. (2017). Psychological scales as predictors of emergency department hospitalizations in suicide attempters. American Journal of Emergency Medicine, 36, 93-99.

Knapik, J. J., Graham, B., Cobbs, J., Thompson, D., Steelman, R., \& Jones, B. H. (2013). A prospective investigation of injury incidence and risk factors among army recruits in combat engineer training. Journal of Occupational Medicine and Toxicology, 8(1), 5. doi:10.1186/17456673-8-5

Kochanek, K. D., Murphy, S. L., Xu, J., \& Arias, E. (2014). Mortality in the United States, 2013. NCHS Data Brief, No. 178.

Levine, D. S., Sripada, R. K., Ganoczy, D., Walters, H., Gorman, L.A., \& Valenstein, M. (2016). Poorer physical health is associated with greater mental health service utilization in a sample of depressed US Army National Guard soldiers. Military Medicine, 181, 803-810.

McCarthy, J. F., Blow, F. C., Ignacio, R. V., Ilgen, M. A., Austin, K. L., \& Valenstein, M. (2012). Suicide among patients in the Veterans Affairs Health System: Rural-urban differences in rates, risks, and methods. American Journal of Public Health, 102(Suppl. 1), S111-S117. doi:10.2105/AJPH.2011.300463

McCarthy, J. F., Bossarte, R. M., Katz, I. R., Thompson, C., Kemp, J., Hannemann, C. M., et al. (2015). Predictive modeling and concentration of the risk of suicide: Implications for preventive interventions in the US Department of Veterans Affairs. American Journal of Public Health, 105, 1935-1942. 
Miller, M., Azrael, D., Barber, C., Mukamal, K., \& Lawler, E. (2012). A call to link data to answer pressing questions about suicide risk among veterans. American Journal of Public Health, 102(Suppl. 1), S20-S22. doi:10.2105/AJPH.2011.300572

Nock, M. K., Stein, M. B., Heeringa, S. G., Ursano, R. J., Colpe, L. J., Fullerton, C. S., et al. (2014). Prevalence and correlates of suicidal behavior among soldiers: Results from the Army Study to Assess Risk and Resilience in Servicemembers (Army STARRS). JAMA Psychiatry, 71, 514-522.

Nyaga, V. N., \& Arbyn, M. (2014). Metaprop: A Stata command to perform meta-analysis of binomial data. Archives of Public Health, 72, 39-49.

Olfson, M., Blanco, C., Wall, M., Liu, S. M., Saha, T. D., Pickering, R. P., \& Grant, B. F. (2017). National trends in suicide attempts among adults in the United States. JAMA Psychiatry, 74, 1095-1103.

Powell, V., Barber, C. W., Hedegaard, H., Hempstead, K., Hull-Jilly, D., Shen, X., et al. (2006). Using NVDRS data for suicide prevention: Promising practices in seven states. Injury Prevention, 12(Suppl. 2), ii28-ii32. doi:10.1136/ip.2006.012443

Rudd, M. D. (2006). Fluid vulnerability theory: A cognitive approach to understanding the process of acute and chronic suicide risk. In T. E. Ellis (Ed.), Cognition and suicide: Theory, research, and therapy (pp. 355-368).

Washington, DC: American Psychological Association.

Shamspour, N., \& Assari, S. (2010). Quality of life in war veterans. In V. R. Preedy \& R. R. Watson (Eds.), Handbook of disease burdens and quality of life measures (pp. 3425-3438). New York: Springer.

Stanley, B., Brown, G. K., Currier, G. W., Lyons, C., Chesin, M., \& Knox, K. L. (2015). Brief intervention and follow-up for suicidal patients with repeat emergency department visits enhances treatment engagement. American Journal of Public Health, 105, 1570-1572. doi:10.2105/AJPH.2015.302656

Ursano, R. J., Kessler, R. C., Stein, M. B., Naifeh, J. A., Aliaga, P. A., Fullerton, C. S., et al. (2015). Suicide attempts in the US Army during the wars in Afghanistan and Iraq, 2004 to 2009. JAMA Psychiatry, 72, 917-926.

U.S. Department of Veterans Affairs. (2018). VA National Suicide Data Report: 2005-2015. Washington, DC: Veterans Health Administration, Office of Mental Health and Suicide Prevention. Retrieved from https://www.mentalhealth.va.gov/docs/datasheets/OMHSP_National_Suicide_Data_Report_ 2005-2015_06-14-18_508-compliant.pdf

Watkins, J. J. (2017). Victor Echo Tango (VET) Support Services Inc. (Unpublished doctoral dissertation). Long Beach: California State University.

Wolfe-Clark, A. L., \& Bryan, C. J. (2016). Integrating two theoretical models to understand and prevent military and veteran suicide. Armed Forces \& Society. Advance online publication. doi:10.1177/0095327X16646645

David S. Wood, PhD, MSW, is assistant professor, School of Social Work, Brigham Young University, 2190 JFSB, Provo, UT 84602; e-mail:d_wood@byu.edu.Bethany M.Wood, MSW, is a social work doctoral student, University of Texas at Austin. Aislinn Watson, MSW, is a social worker, Walker, MI. Devan Sheffield is a medical student, Rocky Vista University, Ivins, UT. Helena Hauter, MSW, CSW, is a clinical social worker, Utah State Hospital, Provo, UT.
Original manuscript received November 29, 2018 Final revision received March 21, 2019

Editorial decision April 3, 2019

Accepted April 5, 2019

Advance Access Publication January 18, 2020 